\title{
Labyrinthe
}

20 | 2005 (1)

La Cognition

\section{Du paralogisme cognitiviste à la médiation sémantique}

Le langage comme enjeu sémiotique

Philippe Lacour

\section{(2) OpenEdition}

\section{Journals}

Édition électronique

URL : http://journals.openedition.org/labyrinthe/757

DOI : $10.4000 /$ labyrinthe. 757

ISSN : 1950-6031

Éditeur

Hermann

Édition imprimée

Date de publication : 20 avril 2005

Pagination : 53-66

\section{Référence électronique}

Philippe Lacour, « Du paralogisme cognitiviste à la médiation sémantique », Labyrinthe [En ligne]

20 | 2005 (1), mis en ligne le 26 juin 2008, consulté le 19 avril 2019. URL : http://

journals.openedition.org/labyrinthe/757 ; DOI : 10.4000/labyrinthe.757

Propriété intellectuelle 


\title{
DU PARALOGISME COGNITIVISTE À LA MÉDIATION SÉMANTIQUE Le langage comme enjeu sémiotique
}

\author{
Philippe LACOUR* \\ placour@netcourrier.com
}

Dans cet article, je cherche à montrer pourquoi l' extension directe des sciences cognitives aux sciences sociales est résistible. Je développe mon argumentation en puisant largement dans l' euvre de deux auteurs français contemporains. L'un, Gilles-Gaston Granger, est épistémologue et philosophe rationaliste. Son cuvre, une ample critique de la raison symbolique, s'avère particulièrement précieuse pour comprendre les particularités respectives des systèmes formels et des langues naturelles, donc pour éviter de les confondre, comme les sciences cognitives sont pourtant tentées de le faire. L'autre, François Rastier, est linguiste et sémioticien. Il insiste, d'une part, sur l' existence d'une zone sémiotique, ontologiquement intermédiaire entre le niveau physique et le niveau des représentations mentales, et, d'autre part, donne à une sémiotique néo-saussurienne le soin de remplir ce rôle charnière. Dans les deux cas, il est remarquable que les réserves touchant les sciences cognitives elles-mêmes, et (du moins chez Rastier) leur extension aux sciences humaines se cristallisent fortement sur le terrain du langage - non celui de la vision, ou de la décision stratégique (le jeu d'échecs par exemple). Cela n'est pas un hasard, tant il est vrai que celui-ci ne saurait être considéré comme l'un des vêtements (parmi d'autres) d'une pensée mentale préexistante, mais comme le milieu, l'élément même de la pensée, dépendant de (mais non réductible à) ses conditions physiques (et neurologiques). Le symbolisme linguistique est le plus important des symbolismes manipulés par

\footnotetext{
* Doctorant en philosophie à l'université d'Aix-Marseille, sous la direction du professeur François Clementz, il occupe actuellement les fonctions de lecteur et de chargé de coopération universitaire à l'université Ca' Foscari de Venise. Ses travaux portent sur la raison pratique, en particulier sur la rationalité et le langage de l'action historique. Il a publié notamment des articles consacrés à l'épistémologie de l'histoire.
} 


\section{Labyrinthe, $n^{\circ} 20$}

l' homme; vouloir en réduire la richesse à sa seule structure formelle est finalement le paralogisme fondamental des sciences cognitives dans leur application au monde humain.

« [...] dans la plupart des cas, en sciences humaines, il est juste de reconnaître qu'on a les mathématiques qu'on mérite, à moins que - pis encore - on ne gaspille des mathématiques luxueuses sur des concepts de deux sous ».

Gilles-Gaston GRANGER ${ }^{1}$

\section{Introduction}

Je vais essayer de soutenir ici que l'idée selon laquelle les sciences humaines et sociales pourraient s'inspirer directement des méthodes des sciences cognitives (sinon de leur objet) relève d'un double paralogisme ${ }^{2}$. La double erreur (formalisation, oubli de la sémantique) est de nature sémiotique, et doit être imputée à une méconnaissance fondamentale des propriétés particulières du symbolisme des langues naturelles. C'est la raison pour laquelle la discussion doit en priorité être portée sur le terrain linguistique, et non sur celui de l'histoire (discipline qui, spontanément, semble la plus à même de résister au formalisme), ou de la psychologie (où le réductionnisme paraît immédiatement devoir conduire le débat). C'est a fortiori parce que toutes les sciences humaines raisonnent dans la langue naturelle, qu'elles sont concernées, sans exception, par «l'aimantation » cognitive.

Les sciences humaines et sociales étudient l'homme et les multiples facettes de son comportement (économique, social, anthropologique, linguistique, politique...); mais elles n'étudient pas la «cognition». Cette différence d'objet est fondamentale et suffirait seule à inspirer la méfiance face à la séduction certaine du paradigme cognitiviste. Certes, on peut bien trouver de nombreux aspects «cognitifs» dans ces disciplines de l'homme, mais c'est au sens très lâche où on suppose, chez l'agent économique ou le locuteur, par exemple, une capacité à réflé-

1. Granger, «Le concept de régulation en linguistique», p. 167.

2. J'aborde le cas d'une inspiration indirecte en conclusion, car il relève somme toute d'une traditionnelle collaboration transdisciplinaire bien comprise. 


\section{Du paralogisme cognitiviste à la médiation sémantique}

chir dont les mécanismes guident «cognitivement» ses choix. Aussi bien y a-t-il peu d'activités humaines auxquelles on ne puisse, en cherchant bien, trouver une dimension cognitive: achat économique, construction d'une phrase, certes, mais aussi course à pied, tir à l'arc, ou cuisine... Cette dilution potentielle (jusqu'au non-sens) de l'objet des sciences cognitives dans les sciences sociales est inscrite dès le départ dans le caractère fortement polysémique de la notion de cognition. Ce «vague» du concept central (mais certains y verront une «richesse»), est la raison pour laquelle l'étude des rapports entre sciences cognitives et sciences sociales ne saurait véritablement commencer par la question de la proximité éventuelle de leur objet.

\section{Le paralogisme de la formalisation}

Mieux vaut lancer l'examen à partir des méthodes, puisque chaque projet scientifique détermine suffisamment la sienne pour qu'elle puisse se prêter à l'épreuve de la comparaison. D'une manière très générale, et indépendamment de la différence entre computationnalisme et connexionnisme ${ }^{3}$, on peut qualifier la méthode cognitiviste de formalisatrice. La formalisation mathématique est évidente dans le modèle computationnaliste (le «comput » correspond à des algorithmes mathématiques), mais elle n'est pas moins présente dans le modèle connexionniste (par volonté de rapprochement avec le cerveau, la neurologie, et les phénomènes d'apprentissage, ce sont des réseaux de neurones formels qui sont constitués ${ }^{4}$ ). Que faut-il entendre exactement par formalisation?

Essentiellement que l'outil de pensée utilisée pour élaborer l'objet «cognition» est le symbolisme mathématique, dont de nombreux penseurs ont tenté de préciser les propriétés spécifiques. Ainsi, pour Gilles-Gaston Granger, la puissance du symbolisme mathématique tient à sa capacité à engendrer des « contenus formels », par abstraction du concret de l'expérience ${ }^{5}$. L'existence virtuelle de tels contenus, due

3. Concernant cette différence, on se reportera à l'article de Pierre Steiner.

4. Voir sur ce point l'entretien avec François Rastier: «Puisque le gène lui-même est conçu comme un programme qui s'applique, même dans ce néo-darwinisme inspiré des sciences de la vie, l'imaginaire logico-formel revient avec insistance, par une mécanisation illusoire du biologique».

5. Sur le rôle d'une dualité fondamentale opération-objet dans la constitution des « contenus formels », Voir Forme, opération, objet, de Gilles-Gaston Granger, chapitres 2 et 3. 
à leur nature formelle, est le garant de leur universalité. Pour autant, cette abstraction se paie en retour d'un abandon, par le symbolisme, des propriétés de référence au monde concret de l'expérience factuelle (ce qui ne veut pas dire que l'on ne peut pas «appliquer» les objets mathématiques virtuels à l'expérience). Au contraire, le symbolisme des langues naturelles, à cause de son ancrage irrémédiablement factuel, ne peut prétendre aux propriétés de précision et d'universalité du symbolisme mathématiques; mais, en contrepartie, cette impuissance est aussi le signe de son immense « richesse » sémantique, beaucoup plus grande et complexe que celle des mathématiques ${ }^{6}$. Or, si le symbolisme mathématique est régi par des conditions de fonctionnement qui sont celles de la logique formelle (qui constitue le canon de la pensée formelle), le symbolisme du langage naturel est, quant à lui, sous-tendu par des conditions plus fondamentales encore, que Gilles-Gaston Granger nomme des conditions «proto-logiques ». Cette asymétrie des conditions est la raison pour laquelle «un système symbolique au sens le plus complet, comme la langue ordinaire, ne se réduit pas à un système formel, essentiellement gouverné par des conditions logiques, bien qu'il puisse être décrit comme tel à un certain niveau ${ }^{7} \gg$.

Tout est dit: la formalisation du langage naturel est possible, mais nécessairement partielle, du fait de la complexité du symbolisme naturel, dont le symbolisme formel est historiquement issu (ce qui ne revient pas à nier les caractéristiques propres du symbolisme formel, que le langage naturel ne possède pas). C'est dans la lignée de cet effort de distinction des propriétés spécifiques des symbolismes, et de dénonciation des transgressions séduisantes, qu'il me paraît instructif d'étudier les réflexions consacrées par Granger touchant à ce que l'on nomme aujourd'hui la «cognition».

Même s'il ne s'est jamais directement préoccupé des «sciences cognitives » comme telles, Granger s'est intéressé très tôt à certains auteurs clé des sciences cognitives (Turing), à la théorie de l'informa-

\footnotetext{
6. Pour une comparaison plus précise de ces deux symbolismes et de leurs rapports, je me permets de renvoyer à mon étude, "Granger et la Critique de la raison symbolique », dont je résume ici les principaux développements.

7. Granger, « Les conditions proto-logiques des langues naturelles » (1989), dans Forme, opération, objet, chapitre 5.
} 


\section{Du paralogisme cognitiviste à la médiation sémantique}

tion et à la naissance de la cybernétique ${ }^{8}$. En fait, cette focalisation sur la «cognition» relève pour lui d'une certaine déviation de la philosophie du langage de son projet véritable (l'étude « des systèmes symboliques en général ${ }^{9}$ ») vers la « philosophie de l'esprit »: « Dans la postérité de la tendance dite "analytique", c'est bien encore le langage et l'exercice du langage qui occupent les philosophes; mais on s'oriente alors davantage vers l'examen des actes de pensée, des croyances, des principes cognitifs sous-tendus par la production et la saisie des sens ${ }^{10}$.»

Face à une telle évolution de la philosophie, les réserves de Granger sont surtout d'ordre méthodologique- outre une première prévention assez classique sur le caractère introspectif de la démarche ${ }^{11}$, il critique surtout le caractère artificiel d'une approche qui, prétendant étudier la connaissance (cognoscere/cognition) délaisse pourtant les réalisations concrètes de cette connaissance pour porter son attention sur des processus invisibles: «[...] s'il s'agit vraiment d'une philosophie de la cognition, pourquoi laisser délibérément de côté les actes de la pensée scientifique décelables à travers les œuvres effectives de la science ${ }^{12}$ ?» De fait, dès lors que la méthode la plus courante consiste à «recourir à une "expérience de pensée" en vue de faire varier les conditions de l'usage du langage, et d'en tirer des conclusions sur des actes de pensée ou leurs présupposés », il n'est pas facile de savoir si les connaissances obtenues relèvent d'une science empirique et objective du langage, de la psycho-linguistique ou d'une interprétation philosophique de l'expérience linguistique. Or, l'enjeu n'est pas mince, puisque aussi bien cette «ambiguïté $[\ldots]$ risque de détourner certains linguistes d'une recherche scientifique, et d'adultérer en contrepartie la philosophie du langage ${ }^{13} »$.

\footnotetext{
8. Voir notamment Pensée formelle et sciences de l'homme, et Essai d'une philosophie du style, de Granger. Mais on trouve aussi de très nombreux textes ultérieurs.

9. Granger, « Le langage dans la philosophie aujourd'hui », p. 70.

10. Id., art. cit., p. 64.

11. Id., art. cit., p. 64 : « En premier lieu, la philosophie de l'esprit ainsi proposée ne va-t-elle pas se confondre avec le produit bâtard d'une introspection, moitié description psychologique - sans garantie de contrôle empirique -, et moitié reconstruction rationnelle? ».

12. Id, art. cit., p. 64. La notion d'œuvre est à ce point fondamentale, dans sa philosophie, que Granger définissait son projet, dans Pensée formelle et sciences de l'homme, comme celui d'une «poiématique », analyse à la fois scientifique, historique et philosophique des œuvres humaines.

13. « Le langage dans la philosophie d'aujourd'hui », art. cit., p. 65.
} 
Que dire de l'intelligence artificielle, fille ou proche cousine du cognitivisme? Essayant de dégager cette notion du «magma syncrétique en fusion - et confusion - des "sciences cognitives" », Granger définit son objet comme "le phénomène de représentation de l'environnement, de stockage et de traitement de la connaissance ${ }^{14} »$. Il remarque aussi comme beaucoup la difficulté qu'il y a à séparer dans cette discipline le savoir et la technique, mais ne la réduit pas pour autant à une simple «technologie », comme Rastier. Il considère en effet que l'on peut distinguer en elle ce qui relève du savoir, et le juger comme tel, d'un point de vue épistémologique. Or, à procéder ainsi, on ne peut que nuancer l'originalité du programme d'intelligence artificielle, dans la mesure où il se ramène en fait au projet beaucoup plus général d'une science de l'homme: «C'est une discipline qui s'occupe de l'étude des faits humains ${ }^{15}$.» Dès lors, on peut dire que, visant le même objet, l'intelligence artificielle rencontre les mêmes difficultés que toutes les sciences humaines, dues à la résistance opposée par leur objet à toute modélisation abstraite: «Prise comme discipline de connaissance scientifique [...], elle rencontre les mêmes difficultés que les autres sciences sociales: difficultés inhérentes à la nature de leur objet, et qui semblent empêcher tout réductionnisme radical, et peutêtre même un réductionnisme modéré ${ }^{16}$. » Rappelons brièvement que ce diagnostic pessimiste sur la science de l'objet humain est constante chez Granger, et toujours expliquée par la complexité du fait humain, sa surdétermination, son caractère symbolique, là où les faits nonhumains peuvent facilement être perçus et conçus comme dépouillés de tout renvoi symbolique (conquête de l'abstraction dont témoigne l'histoire des sciences de la nature), au contraire, le fait humain est immédiatement saisi comme signe et renvoi à autre chose ${ }^{17}$.

Il semble donc que la difficulté soit aussi liée au caractère humain du symbolisme. N'est-ce pas à cela qu'il faut attribuer la difficulté à

14. «the phenomena of representation of the environment, of storage and treatment of knowledge»; voir Granger, « Must a Science of Artificial Intelligence be Necessarily Reductionist?», p. 174.

15. «This is a discipline that [...] does concern itself with the study of human facts», Id., art. cit., p. 181.

16. «Taken as a discipline of scientific knowledge [...], it meets with the same difficulties as does all other social sciences: difficulties inherent in the nature of their object and which seem to rule out any radical reductionism, and perhaps even a moderate one», Id., art. cit., p. 181.

17. Voir «Sur le traitement comme objet des faits humains» (1976), repris dans Forme, opération, objet, p. 260. 
mécaniser la création de symbole? En tout cas, beaucoup plus importante que la collusion entre science et technique en intelligence artificielle, la question cruciale est celle que Granger pose, de façon interronégative, dans la conclusion de son article: "Est-il possible de concevoir des modèles abstraits de l'acte de symbolisation ${ }^{18}$ ?» Le terme important est ici «acte», car il ne s'agit pas d'élaborer un modèle statique, mais de représenter la dynamisation même du symbolisme, sa mise en mouvement, sa créativité. Or, l'activité humaine de symbolisation est particulièrement complexe, et l'intelligence artificielle ne l'aborde qu'avec un schéma «énergétique» (stimulus-réponse) extrêmement pauvre. Pour Granger, ce schéma précède l'activité de symbolisation, et la prépare, mais ne saurait lui-même produire des symboles. La difficulté est donc de savoir si l'activité de symbolisation peut produire sa propre représentation ${ }^{19}$. Or, sans donner une réponse définitivement négative, puisque aussi bien l'épistémologie ne saurait être une discipline prescriptive, force est de constater que l'intelligence artificielle, et les modèles physiques auxquels elle fait appel (que Granger a étudié sous les noms de «cybernétique» et «sémiotique $»^{20}$ ) n'y parviennent pas: «[...] l'intelligence artificielle [...] vient buter sur l'obstacle philosophique radical d'une modélisation de l'activité symbolique ${ }^{21}$.»

On pourrait ici risquer l'hypothèse qu'une telle étude de la cognition, dans la plupart de ses réalisations, mais notamment en intelligence artificielle, relève davantage de la simulation que de la connaissance. Or, même si «l'usage de la simulation dans les sciences contemporaines est sans aucun doute positif ${ }^{22} »$, il faut rappeler contre les assimilations abusives que « la simulation apparaît comme le degré le plus bas d'une échelle dont les degrés supérieurs seraient la compréhension et l'explication ${ }^{23} »$. Même si simuler, dans le domaine scientifique, «c'est déjà expliciter pratiquement un rapport d'opération à objet»

\footnotetext{
18. « Is it possible to conceive abstract models of the act of symbolization?», Id. art. cit., p. 181. 19. Id. art. cit., p. 181: «But modelization, which is an eminently intellectual activity, is already in itself a symbolization at a complex level. Can it, as such, adequately achieve its own representation?». 20. Voir notamment Pensée formelle et sciences de l' homme et Essai d' une philosophie du style, op. cit. 21. [...] artificial intelligence [...] runs up against the radical philosophical obstacle of a modelization of the symbolic activity »; voir Granger, « Must a Science of Artificial Intelligence be Necessarily Reductionist? », art. cit., p. 182.

22. Voir Granger, «Simuler et comprendre », dans Philosophie, langage, science, p. 191.

23. Id., art. cit., p. 191.
} 
appelé à être théorisé ultérieurement, «simuler n'est pas comprendre ${ }^{24}$ ». La formulation algorithmique de processus ${ }^{25}$, quelle que puisse être leur valeur heuristique (par exemple dans le cas des "systèmes experts») est un bon exemple de cette confusion fréquente: «Mais cela se réduit simplement à une transcription directe simplifiée de l'expérience humaine réelle, laquelle, bien qu'elle puisse simuler, n'explique rien $^{26} . »$ Et même si la philosophie ne saurait préjuger a priori de l'échec d'une modélisation de l'activité symbolique elle-même, et se doit de conserver une attitude sceptique ${ }^{27}$, la dénonciation de la confusion, elle, est claire et sans appel : «La tâche du philosophe doit se limiter à exposer et dénoncer l'éternelle tentation consistant à confondre le succès dans la simulation avec l'obtention d'une connaissance scientifique expliquant l'intelligence ${ }^{28}$.»

Non seulement les objections de Granger à l'égard de la cohérence interne du programme cognitif sont dignes de considération, mais sa précieuse différenciation des types de symbolisme fragilise le projet même d'une formalisation exhaustive de la langue naturelle, et rend ainsi caduque l'idée d'une extension directe du programme cognitif aux sciences humaines et sociales. De fait, prendre le langage au sérieux, l'analyser dans son fonctionnement propre conduit nécessairement à lui reconnaitre une place beaucoup plus importante que celle, secondaire, que lui octroie le cognitivisme. Dès lors devient possible un décentrement majeur : penser le projet cognitif à partir du langage, c'est procéder à une culturalisation des sciences cognitives.

24. Id., art. cit., p. 191.

25. Comme le remarque Granger dans son article «Must a Science ... » à la page 179, l'algorithme est un système de règles qui, appliqué aux phénomènes mentaux, laisse complètement irrésolue la notion de méta-règle qu'implique pourtant nécessairement toute notion de règle. Or, «aside from situations of pure calculation, the application of rules is not generally efficient unless controlled by meta-rules whose nature is most often very difficult to formulate ». La promesse du connexionnisme de réduire les méta-règles aux règles (par la notion d' «autopoièse») semble à Granger intéressante mais particulièrement confuse, Id., art. cit., p. 180.

26. « But this boils down to a merely simplified direct transcription of real human experience, which, though it may simulate, explains nothing », Id., art. cit., p. 180.

27. Id, art. cit., p. 182: «Let us recognize however that there does not appear to be anything that needs limit its progress a priori, as long as we are content with measuring that progress in terms of the perfection of the simulation it manages to attain.»

28. «The philosopher's task should be limited here to exposing and denouncing the ever-present temptation to confuse success in simulation with the reaching of scientific knowledge that would be explanatory of intelligence », $I d$., art. cit., p. 182. 


\section{La médiation sémantique contre l'immédiation cognitive}

L'une des erreurs fondamentales du cognitivisme consiste à essayer de découvrir comment «pense » le cerveau humain, en faisant l'hypothèse d'une pensée intérieure fondamentale que la «science» serait susceptible d'atteindre. En suivant cette pente, le langage n'est plus considéré que comme un moyen d'expression, une application parmi d'autres de cette langue de la pensée. D'où la place largement secondaire accordée à la linguistique dans le rapport commandité par la fondation Sloan ${ }^{29}$ en 1978 sur les sciences cognitives. Le célèbre «hexagone cognitif», gage supposé d'une interdisciplinarité harmonieuse, ne réservait en fait à la linguistique qu'un rôle subalterne, organisant au contraire les débats autour des trois pôles de l'ordinateur, du cerveau et de l'esprit, donc en donnant corrélativement la priorité à l'informatique, la logique et la neurophysiologie. À rebours de cette conception qui réduit le langage au rôle de simple véhicule de la pensée, la révolution saussurienne permet de comprendre que la langue n'est pas un instrument mais un milieu naturel, le symbolisme privilégié dans lequel «baigne» la pensée. À ce titre, on ne peut considérer l'analyse du fonctionnement du langage que comme centrale pour l'étude de la cognition.

On sait, en effet, depuis Saussure que le signe linguistique associe un signifiant à un signifié ${ }^{30}$. Ce point connu mérite d'être rappelé pour deux raisons : cela signifie en effet, d'une part, que le sens linguistique n'est pas réductible à une approche exclusivement physicaliste (qui n'en retient que la matière sonore, le signifiant), et, d'autre part, que ce même sens, en tant que signifié, a une valeur diacritique au sein du système de la langue. Cette conception différentielle du signe, si justement célèbre, est pourtant largement méconnue par les analyses (spontanées et savantes) qui confondent sens et référence. Le «sens» de «chien» serait ainsi déictique: le signe désignerait tel quadrupède à poil ras qui agite la queue devant moi, d'où son sens. Pour cette

\footnotetext{
29. Voir le rapport de la fondation Sloan, Sciences cognitives (1978). On retrouve cet hexagone dans l'ouvrage de référence, commandité par cette fondation: Histoire de la révolution cognitive, d'H. Gardner. Pour un exposé critique, voir Simon Bouquet, «De l'hexagramme cognitiviste à une sémiotique de l'interprétation».

30. Voir Écrits de linguistique générale, Bouquet et Engler (éd.), Paris, Gallimard, 2002.
} 
approche «référentielle», la signification d'un concept serait ainsi équivalente à l'étendue des choses dénotées dans le monde. De là, la sémantique cognitive s'est développée par une simple involution mentaliste, préférant substituer un corrélat subjectif au corrélat objectif. Au terme de cette transformation, le sens de «chien» devient la représentation mentale du quadrupède, présente à l'esprit. Cela semble à la fois rigoureux et fidèle à la conception du sens développé par le sens commun, mais c'est une erreur. Le sens linguistique est un signifié, pas un concept ni une représentation psychologique ${ }^{31}$.

C'est donc très largement par un «oubli» de la sémantique du langage naturel que le cognitivisme a pu réunir, en un raccourci saisissant, la chose physique et sa représentation mentale (le sens étant confiné dans la tête, tout en étant garanti par la chose dénotée). Ce n'est pas que l'analyse du langage doive nier l'existence de l'une ou de l'autre. En fait, elle ne fait que plaider en faveur de leur mise en rapport médiate, par l'intermédiaire du signe linguistique, au contraire du cognitivisme, qui les rapproche immédiatement. C'est précisément cette insensibilité à la médiation sémantique opérée par le langage naturel qui fait courir au cognitivisme le risque du réductionnisme. Dès lors en effet que le sens est assimilable à une représentation mentale, pourquoi s'en tenir au dualisme physico-psychique, forcément douteux au regard de la science rigoureuse? Comment ne pas être tenté par l'idée de replier le mental sur le physique, en voyant, par exemple, dans la représentation psychique une survenance de l'activité neurologique, elle-même sous-tendue par des propriétés physiques de la matière? Ce réductionnisme semble en effet suivre une sorte de pente rationnelle, paraissant inciter, ce faisant, la pensée à toujours plus de rigueur. En réalité, l'erreur (très ancienne puisqu'elle précédait la formation de la linguistique) trouve son origine dans une conception erronée du signe linguistique, qui incline insensiblement à ce type de réduction.

Il serait vain de plaider en faveur du dualisme, contre le monisme réductionniste: ce serait là une ligne de défense trop courte. Il faut au contraire faire valoir l'existence non pas de deux mais de trois mondes:

31. François Rastier développe longuement cette originalité de la conception différentielle du sens, par rapport aux approches inférentielle et référentielle, dans Sémantique et recherches cognitives. Pour lui, faire du signifié un concept permet à la philosophie de l'esprit de parler gratuitement du sens linguistique. Voir aussi Lacour, «L'oubli de la sémantique dans le programme cognitiviste », art. cit. 
physique, psychique, mais aussi symbolique. Le symbolique est bien en effet une «couche» de l'Être, dont l'autonomie relative est attestée par la nature double du signe linguistique. Il n'est pas douteux non plus que ce soit cette dualité de nature qui confère au signe linguistique (et par extension au symbolique tout entier) une fonction capitale d'intermédiaire entre le physique et le mental. C'est la raison pour laquelle François Rastier a pu proposer que la sémantique unifiée, une fois son domaine bien établi, reconnu et balisé, s'ouvre, vers l'amont, à l'étude de la perception (qui, bien qu'influencée par la culture, met vraisemblablement en jeu des mécanismes universels), et contribue vers l'aval, à la sémiotique comparée des cultures (chaque sémantique étant relative à une langue particulière) ${ }^{32}$. Reconnaître l'existence (et la prévalence) de la sémantique (le sens comme différence, et non comme référence ou inférence), c'est du même coup conférer une autonomie relative au «monde symbolique», espace situé entre le monde physique et le monde des représentations, et domaine propre des sciences de la culture. C'est sur cette base symbolique (et plus exactement sémantique) solide qu'il devient possible de «culturaliser les sciences cognitives $^{33} \gg$.

\section{Conclusion}

On voit en quoi le second argument (issu de l'œuvre de Rastier) complète le premier (issu des remarques de Granger sur les symbolismes): à chaque fois, c'est à une méconnaissance des propriétés du symbolisme du langage que l'on peut imputer le paralogisme consistant à vouloir étendre directement le programme cognitiviste aux sciences humaines, à commencer par la linguistique ${ }^{34}$. Qui plus est, ce paralogisme ne peut pas être aperçu et identifié tant que l'on ne donne pas au langage naturel la place centrale, et pour ainsi dire matricielle, qui est la sienne dans le fonctionnement de la cognition humaine («centrale» signifie ici primordial, et non unique, exclusif des autres

\footnotetext{
32. Id., art. cit.

33. C'est le projet défendu par François Rastier dans Sémantique et recherches cognitives et Une introduction aux sciences de la culture?

34. Cette extension est rendue possible par l'assimilation du signe linguistique à un symbole formel (les symboles « mentaux » chez Fodor, Andler, etc.).
} 
symbolismes). Faute de percevoir le langage comme un milieu, comme l'élément de la pensée, on en vient à le reléguer au rang de simple instrument de traduction de représentations mentales plus primitive, et à glisser sur la pente dangereuse conduisant immanquablement du formalisme au réductionnisme. Comment ne pas s'inquiéter dès lors, du projet d'extension de l'empire cognitiviste au domaine des sciences humaines, souvent démunies, doutant de leur rationalité, donc proies faciles d'un paradigme dominant? Le risque est d'autant plus grand que toutes prétendent forger des connaissances conceptuelles dans et par le langage naturel ${ }^{35}$, cherchant, qui plus est, à conserver aux hommes dont elles étudient les comportements une marge de liberté, elle-même largement médiatisée par le langage (l'individu «pense», agit librement, réfléchit ses actions très largement dans et par le symbolisme naturel). N'est-ce pas alors à une réduction vertigineuse qu'on assisterait? Pire même, si tant est que la pensée baigne dans le langage comme dans son milieu propre, le risque est grand d'un véritable assèchement du sens, opéré par le monisme formaliste. N'est-ce pas au contraire en donnant toute son amplitude au concept de sens que l'on aura les meilleures chances de saisir l'homme dans ce qu'il a de plus humain: sa créativité symbolique?

Reste que les sciences humaines et sociales, si elles n'ont rien à attendre d'une pure et simple satellisation cognitive, sont parfaitement en droit de s'inspirer indirectement des sciences cognitives ${ }^{36}$. Ce ne serait pas la première fois que le langage naturel ferait intervenir, ponctuellement, des aspects formels, à l'intérieur d'un raisonnement naturel plus vaste, qui en précise les conditions d'application à un cas historique concret et singulier: de ce point de vue, le geste d'utilisation est le même, par-delà la différence des modèles entre, par exemple, économie néo-classique et économie cognitive. Mais il est important de noter que de tels outils formels ne sont jamais auto-suffisants (ils sont toujours requis pour l'explicitation d'une singularité historique précise), et ne doivent intervenir qu'après la prise en considération de

35. Comme le rappelle fortement, et à bon droit, Jean-Claude Passeron, dans son livre décisif, Le Raisonnement sociologique. L'espace non-popperien du raisonnement naturel.

36. En fait, les emprunts pertinents ne concernent pas tant le programme cognitiviste que les résultats de certaines disciplines comme la psycholinguistique, la neurolinguistique, etc. On en reviendrait alors à un cas classique d'interdisciplinarité. 
la sémantique ${ }^{37}$. Ce n'est pas, donc, que les sciences cognitives n'aient aucun intérêt pour les sciences humaines; mais cet intérêt est indirect, médiat, et sa pertinence est toujours subordonnée à celle de la sémantique et du raisonnement naturel, dont le cognitivisme méconnaît en général la conceptualité propre ${ }^{38}$. C'est du fait de cet arrimage indépassable au «raisonnement naturel», au sol factuel du monde historique $^{39}$, que la rationalité des sciences humaines doit faire son deuil d'une rationalité rigoureuse et formelle, et se résoudre au domaine probable de la raison pratique ${ }^{40}$.

\section{BIBLIOGRAPHIE}

BOUQueT Simon, Introduction à la lecture de Saussure, Paris, Payot, 1997. -, «De l'hexagramme cognitiviste à une sémiotique de l'interprétation », dans BOUQUET Simon et RASTIER François, Une introduction aux sciences de la culture?, Paris, Puf, 2002.

Bouquet Simon et RASTIER François (éd.), Une introduction aux sciences de la culture?, Paris, Puf, 2002.

BOUVIER Alban, «Le programme argumentativiste en sociologie et en anthropologie cognitives », dans Nicole Ramognino et Pierre Vergès (dir.), Sciences sociales et cognition sociale, Presses de l'université de Provence, 2005.

37. Le cas est patent en littérature, où l'appel aux schémas mentaux doit suivre et non précéder l'analyse du déploiement, par le texte, de sa signifiance propre. Voir Lacour, «Applying Cognitive Sciences to Literature? ». Pour un point de vue différent, voir l'article de Sylvain Prudhomme.

38. La normativité qu'impose toute réduction n'est pas compatible avec le souci de caractérisation du divers des sciences de la culture.

39. Passeron, op. cit.

40. De ce point de vue, le récent et ambitieux projet argumentativiste d'Alban Bouvier n'est pas sans ambiguïté : il sait en effet rester à bonne distance du réductionnisme (en refusant de se laisser entraîner en deçà de la psychologie cognitive), mais ne prend pas pleinement conscience de sa substance linguistique. Arc-boutant son programme sur une rationalité élargie (théorie du choix rationnel et théorie des croyances rationnelles), dite «cognitive», il ne semble pas tirer pleinement conscience de la « gravité » qui attire nécessairement le raisonnement naturel vers les particularités historiques, puisque cette rationalité fait implicitement allégeance à un infra-individualisme formel (la psychologie cognitive repose elle-même sur une formalisation). Voir Alban Bouvier, «Le programme argumentativiste en sociologie et en anthropologie cognitives ». 


\section{Labyrinthe, $n^{\circ} 20$}

Gardner H., The Mind's New Science. A History of the Cognitive Revolution, New York, Basic Books, 1985 (trad. fr. Histoire de la révolution cognitive, Paris, Payot, 1993).

Granger Gilles-Gaston, Pensée formelle et sciences de l' homme, Paris, Aubier, 1960.

-, Essai d'une philosophie du style, Paris, Armand Colin, 1968.

-, «Le concept de régulation en linguistique» (1978), repris dans Granger 2003, chap. 11.

-, «Must a Science of Artificial Intelligence be Necessarily Reductionnist?», dans E. Agazzi (éd), The Problem of Reductionism in Science, Kluwer Academic Publishers, Netherlands, 1991, p. 173-182. -, Forme, opération, objet, Paris, Vrin, 1994.

-, Philosophie, Langage, Science, Paris, EDP Sciences, 2003.

-, «Le langage dans la philosophie d'aujourd'hui» (article écrit en 1992), Guttorm Floistad (éd.), Language, Meaning, Interpretation, Kluwer Academic Publishers, Netherlands, 2004, p. 45-71.

LACOUR Philippe, «L'oubli de la sémantique dans le programme cognitiviste. Réflexions sur l'oeuvre de François Rastier », http://www.revue-texto.net/Inedits/Inedits.html, 2004.

-, «Granger et la Critique de la raison symbolique », http://formessymboliques.org/article.php3?id_article=130, 2005; article publié avec quelques modifications sur le site: http://www.revue-texto.net

-, «Applying Cognitive Sciences to Literature? », à paraître sur le site http://EspacesTemps.net, 2005.

PASSERON Jean-Claude, Le Raisonnement sociologique. L'espace nonpoppérien du raisonnement naturel, Paris, Nathan, 1991.

Proust Joëlle et SchwarTz Élisabeth (ed.), La Connaissance philosophique, Paris, Puf, 1995.

RASTIER François, Sémantique et recherches cognitives, Paris, Puf, 1991 (seconde édition augmentée 2001).

SAussure F., Écrits de linguistique générale, Bouquet et Engler (éd.), Paris, Gallimard, 2002.

Sloan Foundation, Cognitive Science, 1978, Report of the State of the Art Committee, New-York, 1978. 